\title{
INTEGRAL REPRESENTATION THEOREMS IN TOPOLOGICAL VECTOR SPACES
}

BY

\author{
ALAN H. SHUCHAT( $\left.{ }^{1}\right)$
}

\begin{abstract}
We present a theory of measure and integration in topological vector spaces and generalize the Fichtenholz-Kantorovich-Hildebrandt and Riesz representation theorems to this setting, using strong integrals. As an application, we find the containing Banach space of the space of continuous $p$-normed space-valued functions. It is known that Bochner integration in $p$-normed spaces, using Lebesgue measure, is not well behaved and several authors have developed integration theories for restricted classes of functions. We find conditions under which scalar measures do give well-behaved vector integrals and give a method for constructing examples.
\end{abstract}

In recent years, increasing attention has been paid to topological vector spaces that are not locally convex and to vector-valued measures and integrals. In this article we present a theory of measure and integration in the setting of arbitrary topological vector spaces and corresponding generalizations of the Fichtenholz-Kantorovich-Hildebrandt and Riesz representation theorems. Our integral is derived from the Radon-type integral developed by Singer [20] and Dinculeanu [3] in Banach spaces. This often seems to lend itself to "softer" proofs than the Riemann-Stieltjes-type integral used by Swong [22] and Goodrich [8] in locally convex spaces. Also, although the tensor product notation is convenient in certain places, we avoid the use of topological tensor products as in [22]. Since the spaces need not be locally convex, we must also avoid the use of the Hahn-Banach theorem as in [3], [20], for example.

In $\$ 1$, we develop the basic ingredients of the theory and characterize the continuous linear operators on the space of totally measurable vector functions ( $\mathrm{F}-\mathrm{H}-\mathrm{K}$ theorem). In $\$ \S 2-3$ we characterize the continuous linear functionals and operators on the space $C(Q, E)$ of continuous vector functions with compact support defined on a locally compact space (Riesz theorem). Strong integral representations are obtained, as opposed to the weak representations of Foias, and 1970.

Presented to the Society, January 24, 1970; received by the editors September 20, 4601.

AMS (MOS) subject classifications (1969). Primary 2850, 4625; Secondary 2813,

Key words and phrases. Riesz representation, vector measures, integral representation, containing Banach space, nonlocally convex spaces.

(1) This article is based on the author's dissertation at the University of Michigan and on work supported by the University of Toledo. The author wishes to thank Professor M. S. Ramanujan for his advice and encouragement. 
Singer [7] in Banach spaces and Ulanov [25] and Swong in locally convex spaces. Under additional restrictions on either the spaces or operators, these authors and also Colojoară, Dinculeanu and Marinescu [2] obtain strong representations. Goodrich has a strong representation theorem for the general operator in the locally convex case. After the completion of the dissertation [18] in which most of the present results appeared, the author became aware of the somewhat overlapping work of Edwards and Wayment ([5], [6]), in which strong representation theorems are obtained from a different point of view (similar to that of Goodrich) and with different techniques. In [6], the spaces are locally convex and the authors mention that this restriction can be removed, and in [5], the measures are convex rather than additive. We note that Popescu [15] has defined an integral for set-valued functions in arbitrary spaces.

In $\$ 4$ we specialize to locally bounded spaces and obtain sharper results. The representation theorems are applied to find the containing Banach space [4] of $C(Q, E)$ in this case. In $\$ 5$ we treat scalar measures as operator-valued measures, which yields a Bochner-type integral. Mazur and Orlicz [13], PrzeworskaRolewicz and Rolewicz [16], Gramsch [9] and Vogt [26] discuss the Riemann and Bochner integrals for functions with values in metric linear and locally bounded spaces. The integrals that they define are not, in general, well behaved: Not every continuous function from $[0,1]$ to $l^{p}, 0<p<1$, is Riemann integrable (or Bochner integrable, using Lebesgue measure) and integration is not continuous for the topology of uniform convergence on the space of simple functions. Gramsch and Vogt construct altemate theories, based on topological tensor products, in locally bounded spaces and obtain classes of integrable functions that contain all the holomorphic but not all the continuous functions. Turpin and Waelbroeck [24] generalize these results to locally pseudoconvex spaces and Gramsch [10] has extended some of his results to sequentially complete topological vector spaces. For $l^{p}, 0<p<1$, we obtain a general neccessary condition, that Lebesgue measure fails to satisfy, for the integral to have the desired properties. For any Hausdorff locally bounded space, we obtain a sufficient condition for the integral to be well behaved. Finally, we give a method for constructing a large supply of measures that satisfy this condition for $l^{p}$.

We refer to [12] and [17] for general facts about topological vector spaces.

1. Vector measures. Let $Q$ be a set, $\Sigma$ an algebra of subsets of $Q, E$ and $F$ topological vector spaces (TVS) over either the real or complex field and $\mu$ an additive function on $\Sigma$ with values in $L(E, F)$, the space of continuous linear operators from $E$ to $F$. We wish to integrate $E$-valued functions on $Q$ and have the integral belong to $F$.

A simple function $f: Q \rightarrow E$ is one with a finite number of values, each taken 
on a set in $\Sigma$. For $A \in \Sigma$ and $x \in E$ we denote the characteristic function of $A$ by $c(A)$ and the function $q \rightarrow[c(A)(q)] x$ from $Q$ to $E$ by $c(A) \otimes x$. The class $S(Q, E)$ of simple functions is a vector space, each function $f=\sum_{i=1}^{n} c\left(A_{i}\right) \otimes x_{i}$ is simple and each nonzero simple function has a unique representation in this form, where the $x_{i}$ are distinct and the $A_{i}$ are pairwise disjoint and cover $Q$ $[3$, p. 82]. Such a family of sets, always finite, is called a $\Sigma$-partition of $Q$.

We define the integral of a simple function $f=\Sigma c\left(A_{i}\right) \otimes x_{i}$ by

$$
\int f d \mu=\sum \mu\left(A_{i}\right) x_{i}
$$

and, in particular,

$$
\int c(A) \otimes x d \mu=\mu(A) x \text {. }
$$

The integral is independent of the representation chosen and is a linear transformation from $S(Q, E)$ to $F[3, \mathrm{p} .108]$. Since each simple function is bounded, i.e., its range is a bounded set in $E$, the topology of uniform convergence is a vector topology for $S(Q, E)$. It follows immediately that integration is continuous on $S(Q, E)$ if and only if

for each 0 -neighborhood $V$ in $F$ there is a 0 -neighborhood $U$ in $E$ such that if $x_{1}, \cdots, x_{n}$ belong to $U$ and $A_{1}, \cdots, A_{n}$ is a $\Sigma$-partition of $Q$, then $\Sigma \mu\left(A_{i}\right) x_{i} \in V$.

An additive set function from $\Sigma$ to $L(E, F)$ that satisfies (BSV) is called an $L(E, F)$-valued measure on $\Sigma$ and (BSV) is called the property of bounded semivariation. Only finite additivity is assumed. It is easy to verify that if $E$ and $F$ are Banach spaces then $\mu$ is of bounded semivariation if and only if

$$
\sup \left\|\sum \mu\left(A_{i}\right) x_{i}\right\|_{F}<\infty,
$$

where the supremum is taken over all $\Sigma$-partitions of $Q$ and finite subsets of the closed unit ball in $E$. More generally, if $E$ and $F$ are locally convex spaces then (BSV) is equivalent to Swong's property (V) [22, p. 275]. With the algebraic operations defined in the usual way, the continuity property of (BSV) shows that the $L(E, F)$-valued measures form a vector space.

If $F$ is the scalar field then $\mu: \Sigma \rightarrow E^{\prime}$ is of bounded semivariation if and only if, for some bal anced 0 -neighborhood $U$ in $E$,

$$
\begin{aligned}
\operatorname{Semivar}_{U} \mu & =\sup \left\{\left|\int f d \mu\right|: f \in S(Q, E), f(Q) \subset U\right\} \\
& =\sup \left|\sum\left\langle x_{i}, \mu\left(A_{i}\right)\right\rangle\right|=\sup \sum\left|\left\langle x_{i}, \mu\left(A_{i}\right)\right\rangle\right|
\end{aligned}
$$

is finite, where the last two suprema are taken over all $\Sigma$-partitions of $Q$ and 
elements of $U$ and we use the inner product notation $\langle$,$\rangle for the pairing$ $\left\langle E, E^{\prime}\right\rangle$.

If $X$ is a locally convex space whose topology is given by a family $P$ of seminorms, we say $\mu: \Sigma \rightarrow X$ is of bounded variation if, for each $p \in P$,

$$
\sup \sum p\left[\mu\left(A_{i}\right)\right]<\infty,
$$

taken over all $\Sigma$-partitions of $Q$. Although we will only use this concept for locally convex spaces, the definition can be extended to arbitrary spaces, using pseudometrics [12, p. 52], and Proposition 5 below will remain valid.

For normed $E, \mu: \Sigma \rightarrow E^{\prime}$ is of bounded semivariation if and only if it is of bounded variation, where the usual norm is used for $E^{\prime}$ [23]. For locally convex $E$, (BSV) implies (BV), where $E^{\prime}$ has the strong topology [25, p. 412] and we will show in $\$ \S 2,4$ that these results extend to certain nonlocally convex spaces.

The uniform topology on the vector space of all bounded functions from $Q$ to $E$ is a vector topology and the closure $B(Q, E)$ of $S(Q, E)$ in this space is called the space of totally measurable functions. Since the bounded functions are closed under uniform convergence, $B(Q, E)$ is the space of uniform limits of nets of simple functions and is complete if and only if $E$ is complete. A simple approximation of $f \in B(Q, E)$ is a net of simple functions converging uniformly to $f$. A function from $Q$ to $E$ is $\Sigma$-measurable if the inverse image of each open set in $E$ belongs to $\Sigma$ and totally bounded if its range is a totally bounded set in $E$.

Proposition 1. Every totally measurable function is totally bounded. Every totally bounded $\Sigma$-measurable function is totally measurable and the class of such functions is dense in $B(Q, E)$.

Proof. The first assertion follows from [12, p. 70] and the last assertion is true because every simple function is totally bounded and $\Sigma$-measurable.

Now let $f$ be totally bounded and $\Sigma$-measurable. For each open 0 -neighborhood $U$ in $E$, there are elements $q_{1}, \cdots, q_{n}$ of $Q$ such that the open sets $U_{i}=$ $f\left(q_{i}\right)+U$ cover $f(Q)$. Let $A_{i}=f^{-1}\left(U_{i}\right)$ and $B_{1}=A_{1}, B_{i}=A_{i}-B_{i-1}, i=2, \cdots, n$. Each $B_{i} \subset A_{i}$ and $\left(B_{i}\right)$ is a $\Sigma$-partition of $Q$. The simple function $s=\Sigma_{c}\left(B_{i}\right) \otimes f\left(q_{i}\right)$ has the property that $f(q)-s(q) \in U$ for each $q \in Q$, so the result follows. Note that each such function $f$ has a simple approximation $\left(s_{\alpha}\right)$ such that $s_{a}(Q) \subset f(Q)$ for all $\alpha$. This will be useful in the sequel.

If $F$ is a complete Hausdorff space then the integral defined on $S(Q, E)$ by an $L(E, F)$-valued measure has a unique continuous linear extension to $B(Q, E)$. Thus, under these hypotheses, integration with respect to a vector measure is well defined for totally measurable functions. If $T$ is an operator and $\mu$ an additive set function such that $T f=\int f d \mu$ for all functions $f$ in some class, then $\mu$ repre- 
sents $T$ on that class. The Fichtenholz-Kantorovich-Hildebrandt theorem in this setting is now easy to prove.

Theorem 1. If $F$ is a complete Hausdorff space then $L[B(Q, E), F]$ is isomorphic to the space of $L(E, F)$-valued measures on $\Sigma$. Each operator in $L[B(Q, E), F]$ is uniquely represented on $B(Q, E)$ by the corresponding measure.

Proof. If $\mu$ is an $L(E, F)$-valued measure, let $\pi \mu \in L[B(Q, E), F]$ be the integral determined by $\mu$. By (1), the mapping $\pi$ from the space of measures to $L[B(Q, E), F]$ is one-one. If $T \in L[B(Q, E), F]$ then

$$
\mu(A) x=T[c(A) \otimes x]
$$

defines an additive set function $\mu$ from $\Sigma$ to $L(E, F)$. Since $T$ is continuous and (2) implies that $\mu$ represents $T$ on $S(Q, E), \mu$ is of bounded semivariation and thus a vector measure. By continuity, $\mu$ represents $T$ on all of $B(Q, E)$, $\pi \mu=T$ and $\pi$ is onto. Linearity is obvious, so the result follows.

Corollary. The space of $E^{\prime}$-valued measures is isomorpbic to $B(Q, E)^{\prime}$. Each functional in $B(Q, E)^{\prime}$ is uniquely represented by the measure to which it corresponds.

We note that if no topological properties are assumed, i.e., if $E$ and $F$ are vector spaces, then the proof of Theorem 2 shows that the space of linear transformations from $S(Q, E)$ to $F$ is isomorphic to the space of additive set functions on $\Sigma$ whose values are linear transformations from $E$ to $F$.

Henceforth, $Q$ will denote a locally compact Hausdorff space and $\Sigma$ the Borel class of $Q$, i.e., the $\sigma$-algebra generated by the open sets. A set function $\mu$ from $\Sigma$ to a TVS $X$ is countably additive if $\Sigma \mu\left(A_{i}\right)$ converges to $\mu(A)$ in $X$ for each pairwise disjoint sequence $\left(A_{i}\right)$ of Borel sets with union $A$. We say $\mu$ is regular if, for every Borel set $A$ and 0 -neighborhood $U$ in $X$, there are Borel sets $K$ and $G$ such that

$$
\begin{aligned}
& K \text { is compact, } G \text { is open, } K \subset A \subset G \text { and } \\
& \mu(B) \in U \text { for every Borel set } B \subset G-K .
\end{aligned}
$$

The next few results will be applied in $\$ \S 2-3$. Let $\langle Z, Y\rangle$ be a pairing of vector spaces, $Y$ have the weak topology $\sigma(Y, Z), E$ be a TVS and $\mu$ be a set function from $\Sigma$ to $L(E, Y)$. Each $\theta \in Z$ defines an $E^{\prime}$-valued set function $\mu_{\theta}$ on $\Sigma$ by

$$
\left\langle x, \mu_{\theta}(A)\right\rangle=\langle\theta, \mu(A) x\rangle,
$$

where $A \in \Sigma$ and $x \in E . L(E, Y)$ will have the simple topology of pointwise convergence. 
We will be interested in two particular cases. In $\$ 3$ we let $F$ be a TVS, $Z=F^{\prime}$ and $Y=F_{\sigma}^{\prime \prime}$. (The bidual $F^{\prime \prime}$ is the dual of $F_{\beta}^{\prime}$, which is the dual space $F^{\prime}$ with the strong topology $\beta\left(F^{\prime}, F\right)$. Then $F_{\sigma}^{\prime \prime}$ denotes $F^{\prime \prime}$ with the weak topology $\sigma\left(F^{\prime \prime}, F^{\prime}\right)$.) Thus to $\mu: \Sigma \rightarrow L\left(E, F_{\sigma}^{\prime \prime}\right)$ there corresponds a family of set functions $\mu_{\theta}: \Sigma \rightarrow E^{\prime}, \theta \in F^{\prime}$. In $\$ 2$ we replace $E$ by the scalar field $k, Z$ by $E$ (with a given topology) and $Y$ by $E_{\sigma}^{\prime}$, the dual of $E$ equipped with the weak topology $\sigma\left(E^{\prime}, E\right)$. The space $L\left(k, E_{\sigma}^{\prime}\right)$ is algebraically isomorphic to $E^{\prime}=$ $L(E, k)$ in the obvious way and so, by (4), each $\mu: \Sigma \rightarrow E^{\prime}$ determines a family of scalar-valued set functions $\mu_{x}, x \in E$, defined by

$$
\mu_{x}(A)=\langle x, \mu(A)\rangle
$$

$A \in \Sigma$.

Proposition 2. In the above notation, $\mu$ is additive if and only if eacb $\mu_{\theta}$ is additive and $\mu$ is countably additive (regular) for the simple topology on $L(E, Y)$ if and only if each $\mu_{\theta}$ is countably additive (regular) for $E_{\sigma^{\prime}}^{\prime}$

Proof. The proofs of finite and countable additivity are straightforward and we omit them. The sets

$$
N(x, V)=\{\gamma \in L(E, Y): \gamma x \in V\}
$$

form a local subbase in $L(E, Y)$ as $x$ ranges over $E$ and $V$ ranges over a local subbase in $Y$. The sets

$$
B(\theta, \epsilon)=\{u \in Y:|\langle u, \theta\rangle|<\epsilon\}
$$

form a local subbase in $Y$ as $\theta$ ranges over $Z$ and $\epsilon$ ranges over all positive numbers.

Then $\mu$ is regular if and only if, for each Borel set $A$ and $U=N(x, V)$, where $V=B(\theta, \epsilon)$, there are Borel sets $K$ and $G$ satisfying (3). Also, $\mu(B) \epsilon$ $N(x, V)$ if and only if $\left|\left\langle x, \mu_{\theta}(B)\right\rangle\right|<\epsilon$. Thus $K$ and $G$ satisfy (3) for $\mu$ and $U=N(x, V)$ if and only if they satisfy (3) for each $\mu_{\theta}$ with $U=\left\{z \in E^{\prime}\right.$ :

$|\langle x, z\rangle|<\epsilon\}$, so the proof is complete.

Moreover, as the following result shows, $\mu$ is a measure if and only if each $\mu_{\theta}$ is a measure.

Proposition 3. An additive set function $\mu$ is of bounded semivariation if and only if each $\mu_{\theta}$ is of bounded semivariation.

Proof. We first note that if $s \in S(Q, E)$ then

$$
\left\langle\theta, \int s d \mu\right\rangle=\int s d \mu_{\theta}
$$


for all $\theta \in Z$, which follows directly from the case $s=c(A) \otimes x$ and (4). Now let $s_{a} \rightarrow 0$ in $S(Q, E)$. Then $\int s_{\alpha} d \mu \rightarrow 0$ in $Y$ for $\sigma(Y, Z)$ if and only if $\int s_{a} d \mu_{\theta} \rightarrow 0$ for each $\theta \in Z$. The result follows from the continuity characterization of (BSV).

We note for future reference that (6) implies

$$
\left\langle\theta, \int f d \mu\right\rangle=\int f d \mu_{\theta}
$$

for all $f \in B(Q, E)$, where $\int f d \mu$ belongs to the completion $\hat{Y}$ of $Y$ and the pairing $\langle Z, \hat{Y}\rangle$ is derived in the obvious way from $\langle Z, Y\rangle$.

Since, for the simple topology, $L\left(k, E_{\sigma}^{\prime}\right)$ and $E_{\sigma}^{\prime}$ are isomorphic, we have the following corollary. Weak countable additivity and regularity refer to the topology of $E_{\sigma^{\prime}}^{\prime}$

Corollary. An $E^{\prime}$-valued set function $\mu$ is additive if and only if eacb scalar set function $\mu_{x}$ is additive and $\mu$ is weakly countably additive (regular) if and only if each $\mu_{x}$ is countably additive (regular).

The case of Proposition 3 is more delicate. We may regard $E^{\prime}$ as being either $L(E, k)$ or $L\left(k, E_{\sigma}^{\prime}\right)$, but the notions of bounded semivariation differ because (BSV) depends on the individual topologies involved and not on a topology for the space of mappings. When considering (BSV) for $E^{\prime}$-valued set functions we will always regard $E^{\prime}$ as being $L(E, k)$. With this in mind, we have the following result.

Proposition 4. If an additive $E^{\prime}$-valued set function $\mu$ is of bounded semivariation then each scalar set function $\mu_{x}$ is of bounded variation. The converse is false.

Proof. It suffices to prove that integration with respect to each $\mu_{x}$ is continuous on $S(Q, k)$. If $s$ is a simple scalar function and $x \in E$ then $s \otimes x$ is a simple vector function and

$$
\int s d \mu_{x}=\int s \otimes x d \mu
$$

The assertion follows because if $s_{a} \rightarrow 0$ in $S(Q, k)$ then $s_{a} \otimes x \rightarrow 0$ in $S(Q, E)$.

However, if $E=L^{1}$ (for Lebesgue measure $m$ on the Borel sets of $[0,1]$ ) and $\mu(A)=c(A) \in L^{\infty}=E^{\prime}$ then $\mu$ is finitely additive and $\mu_{x}(A)=\int_{A} x d m$, so each $\mu_{x}$ is of bounded variation. But for any Borel partition of $[0,1]$ into $n$ sets (with nonzero measure), $\Sigma\left\|\mu\left(A_{i}\right)\right\|=n$, so $\mu$ is not of strong bounded variation and, by the remarks following (BSV), not of bounded semivariation.

Since each $\mu_{x}$ is countably additive, the preceding corollary implies that $\mu$ is weakly countably additive. However, it is clear that $\mu$ is not countably additive for the norm topology. This provides a counterexample to an assertion of Singer [20, p. 303]. The corollary to Proposition 5 below shows that Singer's 
assertion becomes true in any TVS if $\mu$ is of strong bounded variation. A similar example can be used to show that the Orlicz-Pettis theorem fails for the weak ${ }^{*}$ and norm topologies of the dual of a Banach space.

We note that if $\mu: \Sigma \rightarrow L(E, Y)$ is additive then the mapping $\theta \rightarrow \mu_{\theta}$ defined by (4) is a linear transformation from $Z$ to the vector space of $E^{\prime}$-valued set functions on $\Sigma$. Finally, it is easy to show that the properties of regularity, finite additivity and countable additivity are preserved in taking linear combinations of set functions. We denote by $M[L(E, Y)]$ the space of all regular countably additive $L(E, Y)$-valued Borel measures on $Q$, using the simple topology on $L(E, Y)$ and the weak topology $\sigma(Y, Z)$ on $Y$. In particular, $M\left(E^{\prime}\right)$ is the space of all weakly regular weakly countably additive $E^{\prime}$-valued Borel measures on $Q$.

As for Banach spaces, problems concerning (BV) can be reduced to problems about positive measures. The proof of the next result is based on [3, pp. 33-36, $42,318]$ and the notation is that of (BV).

Proposition 5. If an additive set function $\mu: \Sigma \rightarrow X$ is of bounded variation then, for each $p \in P$,

$$
\lambda_{p}(A)=\sup \sum p\left[\mu\left(A_{i}\right)\right]
$$

taken over all $\Sigma$-partitions of $A \in \Sigma$, defines a finite positive and finitely additive set function on $\Sigma . \lambda_{p}$ is the least positive increasing superadditive function such that $p[\mu(A)] \leq \lambda_{p}(A)$. Also, $\mu$ is countably additive (regular) if and only if each $\lambda_{p}$ is countably additive (regular).

The corollary below bears on the counterexample in Proposition 4.

Coroll ary. If $E$ and $F$ are TVS, $\mu: \Sigma \rightarrow L(E, F)$ is countably additive for the simple topology and of bounded variation for some topology $t$ of uniform convergence on a family of sets that covers $E$, then $\mu$ is t-countably additive.

Proof. If $\left(A_{i}\right)$ is a pairwise disjoint sequence of Borel sets with union $A$, then since $\mu$ is of bounded variation for $t$, the partial sums of $\Sigma \mu\left(A_{i}\right)$ form a $t$-Cauchy sequence. But $\Sigma \mu\left(A_{i}\right)$ converges to $\mu(A)$ in the simple topology, and the $t$-neighborhoods of 0 are closed for the simple topology, so the result follows from the criterion in $[12, \mathrm{p} .65]$.

2. $C(Q, E)$ and its dual space. Let $C(Q, E)$ denote the space of continuous functions from $Q$ to $E$ with compact support. By Proposition 1, each such function is totally measurable with respect to the Borel sets of $Q$, therefore integrable with respect to each vector-valued Borel measure on $Q$, and we give $C(Q, E)$ the uniform topology of $B(Q, E)$. When $E$ is the scalar field we write $C(Q)$. For each $a \in C(Q)$ and $x \in E$ the function $q \rightarrow a(q) x$ from $Q$ to $E$, denoted by $a \otimes x$, 
belongs to $C(Q, E)$ and the linear span of such functions is isomorphic to the algebraic tensor product $C(Q) \otimes E$.

When $E$ is locally convex $C(Q) \otimes E$ is dense in $C(Q, E)$, and in [19] we show that this is true when $E$ is a complete metrizable TVS with a basis (or satisfies a certain weaker condition) and also when $E$ is an arbitrary TVS and $Q$ has finite covering dimension. It is not known if any space $C(Q, E)$ fails to have this density property and for simplicity, we will assume throughout this article that it holds. Without this assumption, the representation theorems below may only hold for the closure of $C(Q) \otimes E$.

The family of all convex balanced 0-neighborhoods in $E$ is a local base for a locally convex topology on $E$ called the derived locally convex topology. We denote $E$ with this topology by $E_{c} \cdot E_{c}$ has the strongest locally convex topology on $E$ weaker than the original one, $\left(E_{c}\right)^{\prime}=E^{\prime}, E_{c}=E$ if and only if $E$ is locally convex and $E_{c}$ is a Hausdorff space if and only if $E^{\prime}$ separates points of $E[12, \mathrm{p} .109]$. For example, if $E=L^{p}[0,1], 0<p<1$, then $E_{c}$ has the indiscrete topology and if $E=l^{p}, 0<p<1$, then $E_{c}$ has the topology that $l^{p}$ inherits as a subspace of $l^{1}[12$, p. 56]. As far as we know, the following result has appeared only for locally bounded spaces [26, p. 222].

Proposition 6. For every locally convex space $F, L(E, F)=L\left(E_{c}, F\right)$.

Proof. Clearly, $L\left(E_{c}, F\right) \subset L(E, F)$. Conversely, if $T \in L(E, F)$ and $V$ is a convex 0 -neighborhood in $F$, then the convex set $T^{-1} V$ contains a balanced 0 -neighborhood $U$ in $E$ and also its convex hull $\langle U\rangle$. Since $\langle U\rangle$ is balanced, $T$ is also $E_{c}$-continuous.

Theorem 2. $C(Q, E)^{\prime}$ is isomorpbic to $M\left(E^{\prime}\right)$ and each functional in $C(Q, E)^{\prime}$ is uniquely represented on $C(Q, E)$ by the corresponding measure in $M\left(E^{\prime}\right)$.

Proof. By integration, each such $E^{\prime}$-valued measure defines a functional $T \in C(Q, E)^{\prime}$ and the mapping $\pi: M\left(E^{\prime}\right) \rightarrow C(Q, E)^{\prime}$ defined by $\pi \mu=T$ is linear, by the corollary to Theorem 1 . For each $a \in C(Q)$ and $x \in E$,

$$
\int a \otimes x d \mu=\int a d \mu_{x}
$$

obtained by approximating $a$ by simple scalar functions and applying (8). Thus if $\int f d \mu=0$ for all $f \in C(Q, E)$, then each scalar measure $\mu_{x}$ annihilates $C(Q)$. Since $\mu$ is also weakly regular and weakly countably additive, the scalar Riesz representation theorem implies that each $\mu_{x}=0$, so $\mu=0$ and $\pi$ is one-one.

It suffices to show that each $T \in C(Q, E)^{\prime}$ is represented by an appropriate measure $\mu$. For each nonzero $x \in E, C_{x}=\{a \otimes x: a \in C(Q)\}$ is (algebraically) isomorphic to $C(Q)$. The restriction $T_{x}$ of $T$ to $C_{x}$ determines a continuous linear functional on $C(Q)$ and thus a unique regular countably additive scalar 
Borel measure $\mu_{x}$ on $Q$ such that

$$
T[a \otimes x]=\int a d \mu_{x},
$$

for all $a \in C(Q)$. If $x=0$, let $\mu_{x}=0$. Define a set function $\mu$ on the Borel sets of $Q$ by (5). The values of $\mu$ are scalar functions on $E$. The linearity of $T$ and the uniqueness of each $\mu_{x}$ imply that the mapping $x \rightarrow \mu_{x}$ is linear and therefore that $\mu$ is additive. In order to show that $\mu$ is $E^{\prime}$-valued, it suffices to prove that if $x_{a} \rightarrow 0$ in $E$ then the corresponding scalar measures converge to 0 in variation, i.e., the mapping $x \rightarrow T_{x}$ from $E$ to $C(Q)^{\prime}$ is continuous, where $C(Q)^{\prime}$ has the norm topology. Indeed, for each $\epsilon>0$, there is a balanced 0 -neighborhood $U$ in $E$ such that if $f(Q) \subset U$ then $|T f| \leq \epsilon$. For all $a \in C(Q),\|a\| \leq 1$, and large $a$, we have $\left[a \otimes x_{a}\right](Q) \subset U$, so

$$
\left\|T x_{a}\right\|=\sup \left\{\left|T\left[a \otimes x_{a}\right]\right|:\|a\| \leq 1\right\} \leq \epsilon .
$$

In order to prove that $\mu$ is of bounded semivariation, let $\epsilon>0, U$ be a balanced 0 -neighborhood in $E$ as above, $\left(A_{i}\right)$ be a Borel partition of $Q$ and $\left(x_{i}\right)$ be a corresponding subset of $U$. Essentially following the procedure used by Ulanov [25, p. 420], we show that $\left|\Sigma\left\langle x_{i}, \mu\left(A_{i}\right)\right\rangle\right| \leq \epsilon$ by, for any $\delta>0$, combining the regularity of each $\mu_{x_{i}}$ and of the space $Q$ and Urysohn's lemma to obtain functions $a_{i}$ in $C(Q)$ with norm one and pairwise disjoint supports such that

$$
\left|\sum\left\langle x_{i}, \mu\left(A_{i}\right)\right\rangle\right| \leq\left|T\left[\sum a_{i} \otimes x_{i}\right]\right|+\delta
$$

Thus $\mu$ is a weakly regular weakly countably additive $E^{\prime}$-valued Borel measure on $Q$.

The density property of $C(Q, E),(9)$ and (10) imply that $\mu$ represents $T$ on all of $C(Q, E)$, so $T=\pi \mu$ and the proof is complete.

In certain cases the representing measures have stronger properties. In particular, if a representing measure is of strong bounded variation it is also strongly regular and strongly countably additive. Recall that the strong topology $\beta\left(E^{\prime}, E\right)$ of $E_{\beta}^{\prime}$ is determined by the seminorms

$$
p_{M}(\phi)=\sup \{|\phi(x)|: x \in M\}, \quad \phi \in E^{\prime},
$$

where $M$ runs over all weakly bounded sets in $E$.

Proposition 7. Let $\mu: \Sigma \rightarrow E^{\prime}$ be an additive set function of strong bounded variation.

(a) For each weakly bounded set $M$ in $E$ the nonnegative finite set function $\lambda_{M}(A)=\sup \Sigma p_{M}\left[\mu\left(A_{i}\right)\right], A \in \Sigma$, taken over all partitions of $A$ by Borel sets, is additive. 
(b) $\mu$ is strongly countably additive (regular) if and only if $\mu$ is weakly countably additive (regular) if and only if each $\lambda_{M}$ is countably additive (regular).

Proof. By Proposition 5 and its corollary, the only thing left to prove is that weak regularity implies strong regularity, and the proof of this parallels that of Singer in Banach spaces [20, p. 306].

We note that the strong properties mentioned here are equival ent to requiring that the measures $\mu_{x}$ satisfy the corresponding scalar properties uniformly over each weakly bounded set in $E$.

Recall that a measure is only assumed to be (finitely) additive and of bounded semivariation.

Corollary. If every ueakly bounded set in $E$ is bounded then every $E^{\prime}$-valued measure is of strong bounded variation and so the representing measures in Theorem 2 are strongly regular and strongly countably additive. In particular, the result bolds if

(a) the topology of $E$ is weaker than the Mackey topology;

(b) E is locally convex;

(c) every linear functional on $E$ is bounded;

(d) every linear functional on $E$ is continuous.

Proof. If $\mu$ is an $E^{\prime}$-valued Borel measure on $Q$ then, by definition, $\mu$ is of bounded semivariation and so for some balanced 0 -neighborhood $U$, Semivar $U^{\mu}<\infty$. Clearly, $U$ can be replaced by any bounded set in $E$ and so if each weakly bounded set $M$ is bounded then

$$
\sup \sum p_{M}\left[\mu\left(A_{i}\right)\right]=\sup \sum\left|\left\langle x_{i}, \mu\left(A_{i}\right)\right\rangle\right|<\infty,
$$

taken over all Borel partitions of $Q$ and elements of $M$, hence $\mu$ is of strong bounded variation.

Condition (a) is sufficient because then every Mackey bounded set is bounded. Condition (b) is, of course, a special case of (a). Nonlocally convex spaces that satisfy (a) are discussed in [11].

If every linear functional on $E$ is bounded, then it is easy to see that every weakly bounded set $M$ is finite dimensional. Since all finite-dimensional TVS are locally convex [21, p. 35], $M$ is a bounded subset of some subspace of $E$, hence of $E$ itself. Thus (c) is sufficient and, finally, (d) is a special case of (c).

If $E$ is locally bounded but not locally convex then the corollary does not apply. However, we will see in $\$ 4$ that in locally bounded spaces, (BSV) is equivalent to strong bounded variation and so, by Proposition 7 , the representing measures in Theorem 2 are al so strongly countably additive and regular.

We will require the following numerical estimates in $\$ 3$. Let $\mu$ be ap $E^{\prime}$. 
valued Borel measure, $U$ a closed balanced 0 -neighborhood in $E$ for which Semivar $_{U} \mu<\infty$. For each $f \in B(Q, E)$,

$$
N_{U} f=\inf \{b>0: f(Q) \subset b U\}
$$

is finite because $f$ is bounded.

Lemma 1. If $f \in B(Q, E)$ and $N_{U} f \neq 0$ then $f(Q) \subset\left(N_{U} f\right) U$. Thus $N_{U} f \leq 1$ if and only if $f(Q) \subset U$.

Proof. For every $\epsilon>0, f(Q) \subset\left(N_{U} f+\epsilon\right) U$ because there exists $b>0$ such that $N_{U} f<b<N_{U} f+\epsilon$ and $f(Q) \subset b U$. Thus, for each $q \epsilon Q, x_{\epsilon}=\left(N_{U} f+\epsilon\right)^{-1} f(q)$ belongs to $U$ and, since $U$ is closed, $\left(N_{U} f\right)^{-1} f(q)$ al so belongs to $U$.

Proposition 8. If $f \in B(Q, E)$ is $\Sigma$-measurable, then $\left|\int f d \mu\right| \leq\left(N_{U} f\right) \operatorname{Semivar}_{U} \mu$.

Proof. By Propositoin 1, $f$ has a simple approximation $\left(s_{a}\right)$ such that $s_{a}(Q) \subset f(Q)$, hence $N_{U} s_{a} \leq N_{U} f$, for all $\alpha$. Without loss, we may thus assume $f$ is simple. If $N_{U} f \neq 0$, then, by Lemma 1 ,

$$
\left|\int f d \mu\right|=\left(N_{U} f\right)\left|\int\left(N_{U} f\right)^{-1} f d \mu\right| \leq\left(N_{U} f\right) \text { Semivar } \mu \text {. }
$$

If $N_{U} f=0$ then, for all $b>0, f(Q) \subset b U$ and, by the above reasoning, $\left|\int f d \mu\right| \leq$ $b$ Semivar $_{U} \mu$. So $\int f d \mu=0$ and the result follows.

Corollary. If $\mu \in M\left(E^{\prime}\right)$ then

$$
\operatorname{Semivar}_{U} \mu=\sup \left\{\left|\int f d \mu\right|: f \in C(Q, E), N_{U} f \leq 1\right\} \text {. }
$$

Proof. By the proposition, the right side above is no greater than the left. The reverse inequality follows from the uniqueness of the representation in Theorem 2 and (11).

3. Operators on $C(Q, E)$. In this section we represent a continuous linear operator from $C(Q, E)$ to a TVS $F$ whose dual separates points as an $F_{n}^{\prime \prime}$-valued integral by an $L\left(E, F_{n}^{\prime \prime}\right)$-valued Borel measure on $Q . F_{n}^{\prime \prime}$, the bidual $F^{\prime \prime}$ equipped with the natural topology of uniform convergence on equicontinuous sets in $F^{\prime}$, is a locally convex Hausdorff space whose topology is stronger than that of $F_{\sigma^{\prime}}^{\prime \prime}$ If $F$ is a normed space, then $F_{n}^{\prime \prime}$ has the usual norm topology of $F^{\prime \prime}$ [17, p. 143]. By $F_{n}^{\prime \prime}$-valued integral, we mean that the integral is defined as a limit in the space $F_{n}^{\prime \prime}$. Thus this is a strong rather than weak representation. By placing certain additional restrictions on the measure, the representation turns out to be unique. However, because the methods used depend on the pairing $\left\langle F, F^{\prime}\right\rangle$ and the locally convex topologies it determines, the representation does not distinguish the mappings in $L[C(Q, E), F]$ from those in the potentially larger space 
$L\left[C(Q, E), F_{c}\right]$. In general, this is a nontrivial distinction, as the following example shows.

Let $E$ be nonlocally convex and $Q$ compact, so that the function 1 (identically equal to one) belongs to $C(Q)$. The evaluation map $T$ at any fixed point in $Q$ belongs to $L\left[C\left(Q, E_{c}\right), E_{c}\right]$. However, if $x_{a} \rightarrow 0$ in $E_{c}$ but not in $E$ then $1 \otimes x_{a} \rightarrow 0$ in $C\left(Q, E_{c}\right)$ but its image under $T$ diverges in $E$, so $T$ does not belong to $L\left[C\left(Q, E_{c}\right), E\right]$.

If $F^{\prime}$ separates points of $F$ then $F$ may be viewed as a subspace of $F^{\prime \prime}$ and when $F$ is locally convex the topology induced by $F_{n}^{\prime \prime}$ is the original one of $F$.

Proposition 9. If $F$ is a TVS whose dual separates points, then the topology induced on $F$ by $F_{n}^{\prime \prime}$ is that of $F_{c}$.

Proof. Since $F_{c}$ is locally convex, it suffices to show that the topologies of $F$ and $F_{c}$ determine the same equicontinuous sets in $F^{\prime}$. Since $F_{c}$ is weaker than $F$, each $F_{c}$-equicontinuous set is $F$-equicontinuous. Conversely, if $\theta \in F^{\prime}$ maps a balanced 0 -neighborhood in $F$ into a ball $B$ in the scalar field then $\theta$ also maps its convex hull, a 0 - neighborhood in $F_{c}$, into $B$. The result follows.

A family $S$ of $E^{\prime}$-valued measures is of uniformly bounded semivariation if there is a 0 -neighborhood $U$ in $E$ such that

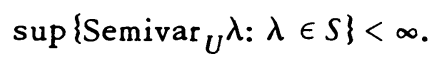

Without loss, we may take $U$ closed and balanced.

Proposition 10. A subset of $M\left(E^{\prime}\right)$ is equicontinuous as a family of functions on $C(Q, E)$ if and only if it is of uniformly bounded semivariation.

Proof. By the corollary to Proposition 8 , Semivar ${ }_{U} \lambda$ can be computed using continuous functions. The necessity of the condition follows easily, using Lemma 1. For sufficiency, if Semivar $U$ is bounded on $S$, then for every $\epsilon>0$, $\lambda \epsilon S$ and $f \epsilon C(Q, E)$ such that $f(Q) \subset \epsilon U$, $\left|\int f d \lambda\right| \leq \epsilon \operatorname{Semivar}_{U} \lambda$, so $S$ is equicontinuous.

Theorem 3. There is an isomorphism of $L[C(Q, E), F]$ into the subspace $M_{n}\left[L\left(E, F_{\sigma}^{\prime \prime}\right)\right]$ of $M\left[L\left(E, F_{\sigma}^{\prime \prime}\right)\right]$ for which

$$
\begin{aligned}
& \text { the mapping } \theta \rightarrow \mu_{\theta} \text { from } F^{\prime} \text { to } M\left(E^{\prime}\right) \text { is } \\
& \text { weakly continuous and takes equicontinu- } \\
& \text { ous sets to families of uniformly bounded } \\
& \text { semivariation. }
\end{aligned}
$$

Each sucb measure is also an $L\left(E, F_{n}^{\prime \prime}\right)$-valued measure and each operator in $L[C(Q, E), F]$ is uniquely represented as an $F_{n}^{\prime \prime}$-valued integral by the corresponding measure. 
Remark. (12) refers to the pairings $\left\langle F^{\prime}, F\right\rangle$ and $\left\langle M\left(E^{\prime}\right), C(Q, E)\right\rangle$. If we replace $F$ by $F_{c}$ (equivalently, if $F$ is locally convex), the isomorphism is onto. It is easy to see that every $L\left(E, F_{n}^{\prime \prime}\right)$-valued measure is an $L\left(E, F_{\sigma}^{\prime \prime}\right)$-valued measure. Since $\hat{F}_{n}^{\prime \prime}$ (the completion of $F_{n}^{\prime \prime}$ ) can be embedded in $\hat{F}_{\sigma}^{\prime \prime}$ with a resultant weakening of its topology $\left[12\right.$, p. 65], the $\hat{F}_{n}^{\prime \prime}$ and $\hat{F}_{\sigma^{\prime}}^{\prime \prime}$ valued integrals determined by such a measure coincide on $B(Q, E)$. Part of the assertion of the theorem is that, for $f \in C(Q, E)$, $\int f d \mu$ belongs to $F$ and converges in $F_{n}^{\prime \prime}$ (not just $\hat{F}_{n}^{\prime \prime}$ ).

Before proving the theorem, we prove the following lemma.

Lemma 2. Each measure in $M_{n}\left[L\left(E, F_{\sigma}^{\prime \prime}\right)\right]$ is an $L\left(E, F_{n}^{\prime \prime}\right)$-valued measure.

Proof. In order to see that $\mu \in M_{n}\left[L\left(E, F_{\sigma}^{\prime \prime}\right)\right]$ is $L\left(E, F_{n}^{\prime \prime}\right)$-valued, let $A$ be a Borel set, $x_{a} \rightarrow 0$ in $E$ and $M \subset F^{\prime}$ be equicontinuous. By (12), there is a closed balanced 0 -neighborhood $U$ in $E$ such that Semivar $_{U}^{\mu}{ }_{\theta}$ is bounded for $\theta \in M$. For each such $\theta$,

$$
\left|\left\langle\theta, \mu(A) x_{a}\right\rangle\right|=\left|\left\langle x_{a}, \mu_{\theta}(A)\right\rangle\right| \leq N_{U}\left[c(A) \otimes x_{a}\right] \text { Semivar }_{U} \mu_{\theta},
$$

and $N_{U}\left[c(A) \otimes x_{a}\right] \rightarrow 0$, so $\mu(A) x_{a} \rightarrow 0$ uniformly on $M$ and thus in $F_{n}^{\prime \prime}$.

Finally, $\mu$ is an $L\left(E, F_{n}^{\prime \prime}\right)$-valued measure, i.e., is of bounded semivariation for $F_{n}^{\prime \prime}$. It suffices to show that if $s_{a} \rightarrow 0$ in $s(Q, E)$, then $\left\langle\theta, \int s_{a} d \mu\right\rangle \rightarrow 0$ uniformly on every equicontinuous set $M \subset F^{\prime}$. But if $U$ is as above,

$$
\left|\left\langle\theta, \int s_{\alpha} d \mu\right\rangle\right|=\left|\int s_{\alpha} d \mu_{\theta}\right| \leq N_{U} s_{a} \operatorname{Semivar}_{U} \mu_{\theta},
$$

so the assertion followis.

The measures in Lemma 2 may not be regular or countably additive for a nice topology on $L\left(E, F_{n}^{\prime \prime}\right)$, as an example of Dinculeanu [3, p. 401] in Banach spaces shows. In this connection, see Proposition 11 below.

Proof of Theorem 3. Let $T \in L[C(Q, E), F]$. We will define a measure $\mu: \Sigma \rightarrow L\left(E, F_{n}^{\prime \prime}\right)$ that satisfies (12) and represents $T$ as follows. We first note that $T^{\prime \prime}: C(Q, E)_{\sigma}^{\prime \prime} \rightarrow F_{\sigma}^{\prime \prime}$ is well defined and continuous, then prove that $T^{\prime \prime} f$ makes sense for $f \in S(Q, E)$ and finally define

$$
\mu(A) x=T^{\prime \prime}[c(A) \otimes x],
$$

for $A \in \Sigma, x \in E$. This defines $\mu(A)$ as a continuous linear mapping from $E$ to $F_{\sigma}^{\prime \prime}$. Next, $\mu \in M\left[L\left(E, F_{\sigma}^{\prime \prime}\right)\right]$ satisfies (12) and represents $T$ as an $F_{\sigma}^{\prime \prime}$-valued integral. Finally, Lemma 2 and the remark imply $T f=\int f d \mu$ as an $F_{n}^{\prime \prime}$-valued integral for all $f \in C(Q, E)$.

We write $M\left(E^{\prime}\right)$ for $C(Q, E)^{\prime}$ and $M\left(E^{\prime}\right)^{\prime}$ for $C(Q, E)^{\prime \prime}$. By [17, pp. 129, $158]$, the existence of the adjoint map $T^{\prime}: F^{\prime} \rightarrow M\left(E^{\prime}\right)$ does not depend on convexity, $T^{\prime}$ is both weakly and strongly continuous and $T^{\prime \prime}: M\left(E^{\prime}\right)^{\prime} \rightarrow F^{\prime \prime}$ exists and is weakly continuous. 
Each $f \in B(Q, E)$ determines, by integration, a linear functional (again denoted by $f)$ on $M\left(E^{\prime}\right)$ and this mapping from $B(Q, E)$ to the algebraic dual of $M\left(E^{\prime}\right)$ is linear, although perhaps not one-one. If $f \in C(Q, E)$ then $f$ is of course strongly continuous, i.e., an element of $M\left(E^{\prime}\right)^{\prime}$, and we will show that this is al so true of simple functions.

It suffices to show that each $c(A) \otimes x$ is strongly continuous and to this end, let $\lambda_{\alpha} \rightarrow 0$ in $M\left(E^{\prime}\right)_{\beta}$ and $\epsilon>0$. Since each $\left(\lambda_{\alpha}\right)_{x}$ is a regular scalar-valued Borel measure, there is a net $\left(a_{\alpha}\right)$ in $C(Q)$ such that $\left\|a_{a}\right\| \leq 1$ and (see (9))

$$
\left|\int c(A) \otimes x d \lambda_{a}\right| \leq\left|\int a_{\alpha} \otimes x d \lambda_{\alpha}\right|+\epsilon \text {. }
$$

By the definition of convergence in $M\left(E^{\prime}\right)_{\beta}$, if the set $\left\{a_{a} \otimes x\right\}$ is weakly bounded in $C(Q, E)$, then the right side of (14) can be made arbitrarily close to $\epsilon$ by taking $\alpha$ arbitrarily large, and this will prove that $c(A) \otimes x$ is strongly con-

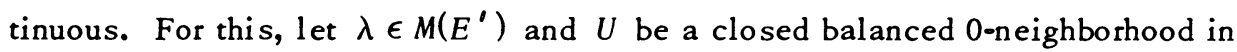
$E$ such that Semivar ${ }_{U} \lambda$ is finite. For each $\alpha$,

$$
\left|\int a_{a} \otimes x d \lambda\right| \leq N_{U}\left(a_{a} \otimes x\right) \operatorname{Semivar}_{U} \lambda \leq N_{U}(1 \otimes x) \text { Semivar }_{U} \lambda,
$$

so $\left\{a_{\alpha} \otimes x\right\}$ is weakly bounded.

It now follows that for $f \in S(Q, E)$, both $T^{\prime \prime} f$ and (13) make sense. Clearly, each $\mu(A)$ is linear, $\mu$ is additive and $T^{\prime \prime} f=\int f d \mu$ for each $f \in S(Q, E)$.

Each $\mu(A) \in L\left(E, F_{\sigma}^{\prime \prime}\right)$ because if $x_{\alpha} \rightarrow 0$ in $E$, then $c(A) \otimes x_{a} \rightarrow 0$ uniformly and thus in $M\left(E^{\prime}\right)_{\sigma}^{\prime}$ and, since $T^{\prime \prime}$ is weakly continuous, $\mu(A) x_{a} \rightarrow 0$ in $F_{\sigma}^{\prime \prime}$.

For the next step, note that, for each Borel set $A, \theta \in F^{\prime}$ and $x \in E$,

$$
\left\langle x, \mu_{\theta}(A)\right\rangle=\left\langle\theta, T^{\prime \prime}[c(A) \otimes x]\right\rangle=\left\langle T^{\prime} \theta, c(A) \otimes x\right\rangle=\int c(A) \otimes x d \lambda=\langle x, \lambda(A)\rangle,
$$

where $\lambda=T^{\prime} \theta \in M\left(E^{\prime}\right)$. Thus the mapping $\theta \rightarrow \mu_{\theta}$ is just $T^{\prime}$, each $\mu_{\theta} \in M\left(E^{\prime}\right)$ and, by Propositions 2 and $3, \mu \in M\left[L\left(E, F_{\sigma}^{\prime \prime}\right)\right] . T^{\prime}$ is weakly continuous and $[17$, p. 130$]$ preserves equicontinuity so, by Proposition 10 and the proof of Proposition 9, $\mu$ satisfies (12). In order to prove that $\mu$ represents $T$ as an $F_{\sigma}^{\prime \prime}$-valued integral, first note that, by (13), $\mu$ represents $T$ " on $S(Q, E)$. If $\left(s_{\alpha}\right)$ is a simple approximation of $f \in C(Q, E)$, then $s_{a} \rightarrow \mathcal{J}$ in $M\left(E^{\prime}\right)_{\sigma}^{\prime}$ Since $T^{\prime \prime}$ is weakly continuous, $\int f d \mu$ belongs to $F^{\prime \prime}$ and equals $T^{\prime \prime} f$. Since the restriction of $T$ " to (the image of) $C(Q, E)$ is $T, T f=\int f d \mu$.

By Lemma 2 and the remark, this part of the proof is complete.

We next show that every measure $\mu \in M_{n}\left[L\left(E, F_{\sigma}^{\prime \prime}\right)\right]$ determines, by integration, an operator in $L\left[C(Q, E), F_{c}\right]$. By Lemma 2 and the remark, $T$ belongs to $L\left[C(Q, E), \hat{F}_{n}^{\prime \prime}\right]$, so by Proposition 9, it suffices to show that, for each $f \in C(Q, E)$, $\int f d \mu$ belongs not only to $\hat{F}_{n}^{\prime \prime}$ but actually to $F$. It thus suffices to show that $\int f d \mu$ is continuous on $F_{\sigma}^{\prime}$ and to this end, let $\theta_{a} \rightarrow 0$ in $F_{\sigma}^{\prime}$. Since $\theta \rightarrow \mu_{\theta}$ 
is weakly continuous, (7) implies that $\int f d \mu \theta_{\alpha} \rightarrow 0$ and so $T \in L\left[C(Q, E), F_{c}\right]$. Moreover, the measure obtained from $T$ by (13) is precisely $\mu$, because the representation is unique. Indeed, if $\mu \in M\left[L\left(E, F_{\sigma}^{\prime \prime}\right)\right]$ satisfies (12) and $\int f d \mu=0$ for all $f \in C(Q, E)$, then, for all $\theta \in F^{\prime}, \int f d \mu_{\theta}=0$. By the uniqueness in Theorem 2, each $\mu_{\theta}=0$ and so $\mu=0$.

Thus the range of the mapping $\pi: L[C(Q, E), F] \rightarrow M\left[L\left(E, F_{\sigma}^{\prime \prime}\right)\right]$ defined by (13) is contained in the subspace $M_{n}\left[L\left(E, F_{\sigma}^{\prime \prime}\right)\right]$ satisfying (12), and if we define $\pi$ on the larger space $L\left[C(Q, E), F_{c}\right]$, then $\pi$ is onto. Obviously, $\pi$ is one-one and, since the taking of adjoints is linear, $\pi$ is linear. So $\pi$ is an isomorphism and the proof of the theorem is complete.

Corollary 1. If $F_{c}$ is semireflexive then the representing measures in Theorem 3 are $L\left(E, F_{c}\right)$-valued measures and the corresponding integrals converge in $F_{c}$.

Corollary 2. In addition to the bypotheses of Theorem 3, let $E^{\prime}$ separate points of $E$ and $F_{c}$ be quasicomplete. Then the operators in $L\left[C(Q, E), F_{c}\right]$ that are weakly compact in $L\left[C(Q, E)_{c}, F_{c}\right]$ correspond to the $L\left(E, F_{c}\right)$-valued representing measures for which the mapping $\theta \rightarrow \mu_{\theta}$ is continuous for $F_{\sigma}^{\prime}$ and $M\left(E^{\prime}\right)$ with the weak topology $\sigma\left(M\left(E^{\prime}\right), M\left(E^{\prime}\right)^{\prime}\right)$.

Proof. Note that Proposition 6 implies $L\left[C(Q, E), F_{c}\right] \subset L\left[C(Q, E){ }_{c}, F_{c}\right]$. This corollary is a standard application of [1, p. 89] as in Swong [22, p. 285], once we observe that if $E^{\prime}$ separates points of $E$ then $M\left(E^{\prime}\right)$ separates points of $C(Q, E)$, since $C(Q, E)_{c}$ must be a Hausdorff space. Indeed, since $E^{\prime}$ separates points of $E, E_{c}$ is a Hausdorff space and so is the locally convex space $C\left(Q, E_{c}\right)$, whose dual $M\left(E_{c}^{\prime}\right)$ thus al so separates points. Also, $C(Q, E) \subset$ $C\left(Q, E_{c}\right), M\left(E_{c}^{\prime}\right) \subset M\left(E^{\prime}\right)$ and if $f \in C(Q, E)$ and $\mu \in M\left(E_{c}^{\prime}\right)$ then $\int f d \mu$ has the same meaning if we regard $\mu$ as being in $M\left(E^{\prime}\right)$. Thus $M\left(E^{\prime}\right)$ separates points of $C(Q, E)$ and the proof is complete.

Proposition 11. If a representing measure $\mu \in M_{n}\left[L\left(E, F_{\sigma}^{\prime \prime}\right)\right]$ is of bounded variation for the topology of bounded convergence on $L\left(E, F_{n}^{\prime \prime}\right)$, then $\mu$ is countably additive and regular for that topology.

Proof. The topology of $L\left(E, F_{n}^{\prime \prime}\right)$ is given by the seminorms

$$
p_{M B}(u)=\sup \left\{p_{M}(u x): x \in B\right\}
$$

where $B \subset E$ is bounded, $M \subset F^{\prime}$ is equicontinuous and

$$
p_{M}(\phi)=\sup \left\{|\langle\theta, \phi\rangle|: \theta \in F^{\prime}\right\}
$$

is the corresponding seminorm for $F_{n}^{\prime \prime}$. Let $\lambda_{M B}$ be the corresponding positive measure. 
For countable additivity, we note that if $\left(A_{i}\right)$ is a sequence of pairwise disjoint Borel sets with union $A$, then the partial sums of $\Sigma \mu\left(A_{i}\right)$ form a Cauchy sequence in $L\left(E, F_{n}^{\prime \prime}\right)$ for the topology of bounded convergence. Moreover, it is easy to see that the 0 -neighborhoods determined by the $p_{M B}$ are closed for the restriction to $L\left(E, F_{n}^{\prime \prime}\right)$ of the simple topology of $L\left(E, F_{\sigma}^{\prime \prime}\right)$. Since $\Sigma \mu\left(A_{i}\right)$ converges to $\mu(A)$ in the latter topology, the result follows as in the corollary to Proposition 5.

For regularity, fix some $M$ and $B$, let $A$ be a Borel set and $\epsilon>0$. Let $A_{1}$, $\cdots, A_{n}$ be a Borel partition of $A$ such that

$$
\lambda_{M B}(A)-\sum p_{M B}\left[\mu\left(A_{i}\right)\right]<\epsilon,
$$

$x_{1}, \cdots, x_{n} \in B$ such that each

$$
p_{M B}\left[\mu\left(A_{i}\right)\right]-p_{M}\left[\mu\left(A_{i}\right) x_{i}\right]<\epsilon / n,
$$

and $\theta_{1}, \ldots, \theta_{n} \in M$ such that each

$$
p_{M}\left[\mu\left(A_{i}\right) x_{i}\right]-\left|\left\langle\theta_{i}, \mu\left(A_{i}\right) x_{i}\right\rangle\right|<\epsilon / n
$$

Suppress the indices on the vectors and recall that each $\left(\mu_{\theta}\right)_{x}$, defined by (4) and (5), is a regular scalar measure, so that there are compact sets $K_{i} \subset A_{i}$ such that

$$
\left|\left(\mu_{\theta}\right)_{x}\left(A_{i}\right)\right|-\left|\left(\mu_{\theta}\right)_{x}\left(K_{i}\right)\right|<\epsilon / n \text {. }
$$

Let $K=\bigcup K_{i}$ and paste these relations together (with certain additional obvious ones as in $[20, \mathrm{p} .306])$ to obtain

$$
\lambda_{M B}(A)-\lambda_{M B}(K)<4 \epsilon .
$$

The existence of a suitable open set $G$ containing $A$ is obtained by duality, and the proof is complete.

4. Vector measures in locally bounded spaces. Recall that a locally bounded space is a TVS $E$ with a loçal base of bounded sets and, by a theorem of Rolewicz [26, p. 220], that the topology of a Hausdorff locally bounded space is always given by a $p$-norm, $0<p \leq 1$. A $p$-norm is a nonnegative positive definite subadditive functional \|\| that is $p$-homogeneous, i.e., $\|b x\|=|b|^{p}\|x\|$ for all scalars $b$ and vectors $x$. If no 1 -norm exists then $E$ is not locally convex. In this section, $E$ and $F$ and $p$ - and $q$-normed spaces, respectively, $0<p, q \leq 1$, and $F^{\prime}$ separates points of $F$. It is easy to see, by mimicking the Banach space argument, that an additive $L(E, F)$-valued set function $\mu$ is of bounded semivariation if and only if $\left(\mathrm{BSV}^{\prime}\right)$ holds, where we use the $p$ - and $q$-norms instead of norms. Denote this supremum by Semivar $\mu$. Also, $L(E, F)$ has a naturally defined $q$-norm, namely

$$
\|T\|=\sup \left\{\|T x\|_{F}:\|x\|_{E} \leq 1\right\}
$$


corresponding to the topology of bounded convergence. It is easy to see that (BV) for this $q$-norm implies (BSV'). Also, (15) defines a norm for $E^{\prime}$.

Proposition 12. The derived locally convex topology of $E$ is a seminorm topology, given by

$$
\|x\|_{1}=\sup \left\{|T(x)|: T \in E^{\prime},\|T\| \leq 1\right\} .
$$

$E_{c}$ is a normed space if and only if $E^{\prime}$ separates points of $E$.

Proof. Clearly, \|\|$_{1}$ is a seminorm and is a norm if and only if $E^{\prime}$ separates points. The inequality $|T(x)| \leq\|T\|\|x\|^{1 / p}, T \in E^{\prime}$, implies

$$
\|x\|_{1} \leq\|x\|^{1 / p} \text {, }
$$

so the \|\|$_{1}$-topology is weaker than the p-norm topology and $\left(E,\|\|_{1}\right)^{\prime} \subset E^{\prime}$. Conversely, if $T \in E^{\prime},\|T\| \leq 1$, then by (16), $T$ is \|\|$_{1}$-continuous, so $\left(E,\|\|_{1}\right)^{\prime}=E^{\prime}$. Thus $\left(E,\|\|_{1}\right)$ has the Mackey topology for $\left\langle E, E^{\prime}\right\rangle$ and, by the Mackey-Arens theorem, must be $E_{c}$.

Corollary 1. The norm on $E^{\prime}$ given by (15) equals

$$
\|T\|=\sup \left\{|T(x)|:\|x\|_{1} \leq 1\right\} .
$$

Proof. The closed unit ball $B_{1}$ in $E_{c}$ is the bipolar of the closed unit ball $B$ in $E$ (for the p-norm) for the pairing $\left\langle E, E^{\prime}\right\rangle$ and $B$ is balanced, so, by the bipolar theorem, $B_{1}$ is the weak closure in $E$ of the convex hull of $B$. Thus the suprema in (18) and (15) are equal.

Comllary 2. For an $E^{\prime}$-valued additive set function $\mu,(B S V)$ and strong bounded variation are equivalent. Moreover, Semivar $\mu$ is finite and equals the supremum Var $\mu$ in $(B V)$, where we use the norm on $E^{\prime}$.

Proof. By the preceding results, the strong topology of $E^{\prime}$ is the norm topology. The proof is now similar to that in Banach spaces [3, p. 54].

Note that $B(Q, E)$ is a $p$-normed space with

$$
\|f\|=\sup \left\{\|f(t)\|_{E}: t \in Q\right\}
$$

so $C(Q, E)^{\prime}$ is a normed space. Also, the variation is a norm on $M\left(E^{\prime}\right)$, as for Banach spaces. As usual, we assume that $C(Q, E)$ has the density property.

Theorem 4. $C(Q, E)^{\prime}$ is isometrically isomorpbic to $M\left(E^{\prime}\right)$ and $M\left(E^{\prime}\right)$ is the space of all strongly regular strongly countably additive $E^{\prime}$-valued Borel measures of strong bounded variation.

Proof. By Corollary 2 above and Proposition $7, M\left(E^{\prime}\right)$ is the space described. 
It suffices to show that the isomorphism in Theorem 2 is an isometry, and this now follows from Lemma 1 and the corollary to Proposition 8.

For the operator case, note that $F_{n}^{\prime \prime}$ is a normed space and that Semivar $\mu$ is a norm on $M_{n}\left[L\left(E, F_{\sigma}^{\prime \prime}\right)\right]$. Also, recall that $L[C(Q, E), F]$ is a q-normed space and is contained in the normed space $L\left[C(Q, E), F_{c}\right]$.

Theorem 5. $L\left[C(Q, E), F_{c}\right]$ is isometrically isomorphic to $M_{n}\left[L\left(E, F_{\sigma}^{\prime \prime}\right)\right]$ and if $\mu$ represents $T$ then

$$
\|T\|=\sup \left\{\operatorname{Var} \mu_{\theta}:\|\theta\|_{F^{\prime}} \leq 1\right\}=\operatorname{Semivar} \mu .
$$

$L[C(Q, E), F]$ is isomorphic to the subspace of measures in $M_{n}\left[L\left(E, F_{\sigma}^{\prime \prime}\right)\right]$ for which the $q-$ norm

$$
\|T\|_{q}=\sup \left\{\left\|\int f d \mu\right\|_{F}: f \in C(Q, E),\|f\| \leq 1\right\}
$$

is finite, and we have Semivar $\mu \leq\|T\|_{q}^{1 / q}$.

Proof. First, for $f \in C(Q, E)$,

$$
\|T f\|_{F_{c}}=\left\|\int f d \mu\right\|_{F_{n}^{\prime \prime}}=\sup \left\{\left|\left\langle\theta, \int f d \mu\right\rangle\right|:\|\theta\|_{F^{\prime}} \leq 1\right\} .
$$

So, by the corollary to Proposition 8 and Corollary 2 above,

On the other hand, clearly

$$
\begin{aligned}
\|T\| & =\sup \left\{\left|\int f d \mu_{\theta}\right|:\|f\| \leq 1,\|\theta\| \leq 1\right\} \\
& =\sup \left\{\operatorname{Var} \mu_{\theta}:\|\theta\| \leq 1\right\} .
\end{aligned}
$$

$$
\left\|\sum \mu\left(A_{i}\right) x_{i}\right\|_{F_{n}^{\prime \prime}}=\sup \left\{\left|\sum \mu_{\theta}\left(A_{i}\right) x_{i}\right|:\|\theta\|_{F^{\prime}} \leq 1\right\},
$$

so that $\|T\|=$ Semivar $\mu$.

Also, $T \in L[C(Q, E), F]$ if and only if its q-norm satisfies (19), and, by (17),

$$
\|T f\|_{F_{c}} \leq\|T f\|_{F}^{1 / q},
$$

so $\|T\| \leq\|T\|_{q}^{1 / q}$ and the proof is complete.

We say $T \in L[C(Q, E), F]$ is dominated if there is a finite positive regular Borel mea sure $\nu$ such that

$$
\|T f\|_{F_{c}} \leq \int\|f\|^{1 / p} d \nu
$$

for all $f \in C(Q, E)$, where $\|f\|^{1 / p}$ denotes the function $t \rightarrow\|f(t)\|_{E}^{1 / p}$. We use the same notation for $f \in B(Q, E)$. This concept was introduced by Dinculeanu for Banach spaces (see [3, p. 416] for historical references) and has been applied by Pietsch [14, Satz 2.3.4] and Swartz [27] in the study of absolutely summing operators. 
Let $\lambda$ denote the total variation of $\mu$ for the norm on $L\left(E, F_{n}^{\prime \prime}\right)$, as defined in Proposition 5. The completion $\hat{F}_{c}$ of $F_{c}$ is the containing Banach space discussed below.

Theorem 6. The representing measure $\mu$ corresponding to $T \in L[C(Q, E), F]$ is of bounded variation in $L\left(E, F_{n}^{\prime \prime}\right)$ if and only if $T$ is a dominated operator. In this case, $\mu$ is a countably additive regular $L\left(E, \hat{F}_{c}\right)$-valued measure of bounded variation (all in the norm of $L\left(E, \hat{F}_{c}\right)$ ), the integral converges in $\hat{F}_{c}$ and, for all $f \in B(Q, E)$,

$$
\left\|\int f d \mu\right\|_{F_{n}^{\prime \prime}} \leq \int\|f\|^{1 / p} d \lambda .
$$

Proof. If $\mu \in M_{n}\left[L\left(E, F_{\sigma}^{\prime \prime}\right)\right]$ represents $T \in L[C(Q, E), F]$ and is of bounded variation in $L\left(E, F_{n}^{\prime \prime}\right)$ then for $s=\Sigma c\left(A_{i}\right) \otimes x_{i}$ in $S(Q, E)$,

$$
\left\|\int s d \mu\right\|_{F_{n}^{\prime \prime}} \leq \sum\left\|\mu\left(A_{i}\right)\right\|\left\|x_{i}\right\|^{1 / p} \leq \sum\left\|x_{i}\right\|^{1 / p} \lambda\left(A_{i}\right)=\int\|s\|^{1 / p} d \lambda .
$$

If $\left(s_{\alpha}\right)$ is a simple approximation of $f \in B(Q, E)$, then $\left\|s_{a}\right\|^{1 / p} \rightarrow\|f\|^{1 / p}$ uniformly, so (21) holds and, in particular, (20) holds with $\nu=\lambda$. By Proposition 11, $\lambda$ is finite, countably additive and regular, so $T$ is dominated.

Conversely, if $T$ is dominated it suffices to show that $\lambda \leq \nu$. Given a Borel partition $A_{1}, \ldots, A_{n}$ of $A \in \Sigma$ and corresponding vectors $x_{i} \in E, \theta_{i} \in F^{\prime}$ with $\left\|x_{i}\right\| \leq 1,\left\|\theta_{i}\right\| \leq 1$, we must show

$$
\sum\left|\left\langle\theta_{i}, \mu\left(A_{i}\right) x_{i}\right\rangle\right| \leq \nu(A) .
$$

Let $\epsilon>0$ and, without loss, take $\left\|x_{i}\right\|=1$. There are functions $a_{i} \in C(Q), 0 \leq$ $a_{i} \leq c\left(A_{i}\right)$, such that (we suppress the indices on the vectors)

Then

$$
\left|\int c\left(A_{i}\right) d\left(\mu_{\theta}\right)_{x}\right| \leq\left|\int a_{i} d\left(\mu_{\theta}\right)_{x}\right|+\epsilon / n
$$

$$
\begin{aligned}
\sum\left|\left\langle\theta_{i}, \mu\left(A_{i}\right) x_{i}\right\rangle\right| & \leq \sum\left|\left\langle\theta_{i}, \int a_{i} \otimes x_{i} d \mu\right\rangle\right|+\epsilon \\
& \leq \sum\left\|\int a_{i} \otimes x_{i} d \mu\right\|+\epsilon \\
& \leq \sum \int\left\|a_{i} \otimes x_{i}\right\|^{1 / p} d \nu+\epsilon=\int|a| d \nu+\epsilon \\
& \leq \nu(A)+\epsilon,
\end{aligned}
$$

and $\epsilon$ is arbitrary, so (22) follows.

Now suppose $\mu$ is of bounded variation. By Proposition 11, it suffices to show each $\mu(A) x \in \hat{F}_{c}$. We first show there is a net $\left(a_{a} \otimes x\right)$ in $C(Q, E)$ such that $\int a_{a} \otimes x d \mu \rightarrow \int c(A) \otimes x d \mu$ in $F_{n}^{\prime \prime}$. Since $\lambda$ is regular, for each $\epsilon>0$ there exists $a \in C(Q), 0 \leq a \leq c(A)$, such that $\int|c(A)-a| d \lambda<\epsilon$. Then 


$$
\begin{aligned}
\left\|\int[c(A)-a] \otimes x d \mu\right\|_{F_{n}^{\prime \prime}} & \leq \int\|[c(A)-a] \otimes x\|^{1 / p} d \lambda \\
& =\|x\|^{1 / p} \int|c(A)-a| d \lambda \\
& <\epsilon\|x\|^{1 / p}
\end{aligned}
$$

so such a net exists.

The seminorm $f \rightarrow\left\|\int f d \mu\right\|$ (norm in $F_{n}^{\prime \prime}$ ) determines a vector topology on $B(Q, E)$ in which $f_{a} \rightarrow 0$ if and only if $\int f_{a} d \mu \rightarrow 0$ in $F_{n}^{\prime \prime}$. Then $T: C(Q, E) \rightarrow F_{c}$ is continuous for this topology and has a unique continuous linear extension, with values in $\hat{F}_{c}$, to the closure of $C(Q, E)$. The above remarks imply that $C(Q, E)$ is dense in $B(Q, E)$, and $T f=\int f d \mu$ for $f \in B(Q, E)$. Since $T[c(A) \otimes x]=\mu(A) x$, the proof is complete.

In a recent paper, Duren, Romberg and Shields [4] discussed the notion of the containing Banach space of a $p$-normed space (whose dual separates points). The remainder of this section is devoted to finding the containing Banach space of $C(Q, E)$.

If $E^{\prime}$ separates points of $E$, then, by Proposition $12, E_{c}$ is a normed space so its completion $\hat{E}_{c}$ is a Banach space with the same dual as $E$. $\hat{E}_{c}$ is called the containing Banach space of $E . C(Q, E)$ is a p-normed space and, by the proof of Corollary 2 of Theorem $3, M\left(E^{\prime}\right)$ separates points of $C(Q, E)$, so $C(Q, E)$ has a containing Banach space. The containing Banach space has the topology of the natural bidual.

In general, if $X$ is a TVS, a function $f: Q \rightarrow X$ vanishes at infinity if, for each 0 -neighborhood $U$ in $X$, there is a compact set $K \subset Q$ such that $f(Q-K) \subset$ $U$. The continuous functions that vanish at infinity form a vector space $C_{0}(Q, X)$ and, since they are bounded, the uniform topology is a vector topology.

Proposition 13. If $X$ is complete, then $C_{0}(Q, X)$ is the completion of $C(Q, X)$.

Proof. If $\left(f_{\alpha}\right)$ is a Cauchy net in $C_{0}(Q, X)$, then, since $X$ is complete, $\left(f_{a}\right)$ converges uniformly to a bounded continuous function $f$ and we will show $f$ vanishes at infinity. If $U$ is a 0 -neighborhood in $X$, then there is a 0 -neighborhood $V$ such that $V+V \subset U$ and some a such that $\left(f-f_{a}\right)(Q) \subset V$. There is a compact set $K \subset Q$ such that $f_{a}(Q-K) \subset V$ and then

$$
f(Q-K) \subset\left(f-f_{\alpha}\right)(Q-K)+f_{\alpha}(Q-K) \subset V+V \subset U .
$$

Thus $f$ vanishes at infinity and $C_{0}(Q, X)$ is complete.

Now let $f \in C_{0}(Q, X)$ and $U$ be a 0 -neighborhood in $X$. Let $V$ be as above, $K \subset Q$ a compact set such that $f(Q-K) \subset V$ and $a \in C(Q), 0 \leq a \leq 1$, be identically one on $K$. The function af defined by $[a f](t)=a(t) f(t)$ belongs to $C(Q, X)$ 
and agrees with $f$ on $K$. For $t \in Q-K,[f-a f](t) \in V+V \subset U$, so $[f-a f](Q) \subset$ $U$. Thus $C(Q, X)$ is dense in $C_{0}(Q, X)$ and the proof is complete。

Theorem 7. The containing Banach space of $C(Q, E)$ is $C_{0}\left(Q, \hat{E}_{c}\right)$.

Proof. We first show that the derived locally convex topology of $C(Q, E)$ is the weaker norm topology it inherits as a subspace of $C\left(Q, E_{c}\right)$. Since $C\left(Q, E_{c}\right)^{\prime}$ $=M\left(E_{c}^{\prime}\right)$ and the inherited topology makes $C(Q, E)$ a Mackey space, it suffices to show that $M\left(E^{\prime}\right)=M\left(E_{c}^{\prime}\right)$. Since $E^{\prime}=E_{c}^{\prime}$ as sets, we need only show that (BSV) has the same meaning for $E$ and $E_{c}$, and this follows from Corollaries 1 and 2 of Proposition 12.

It now suffices to show that $C(Q, E)$ is dense in $C_{0}\left(Q, \hat{E}_{c}\right)$. Since $E$ is dense in $\hat{E}_{c}, C(Q) \otimes E$ is dense in $C(Q) \otimes \hat{E}_{c}$ for the uniform topology. But $C(Q) \otimes \hat{E}_{c}$ is dense in $C\left(Q, \hat{E}_{c}\right)$, since the density property holds for locally convex spaces, so the result follows.

5. Scalar measures as vector measures. If $\mu$ is a finite positive Borel measure on $Q$, then by treating the values of $\mu$ as multiplication operators on a TVS $E$ we may regard $\mu$ as an additive $L(E, E)$-valued set function. It is easy to see that, when $E$ is locally convex, (BSV) is satisfied, so $\mu$ is a: vector measure, and $\mu$ is countably additive for the topology of bounded convergence on $L(E, E)$. In particular, if $E$ is a Banach space we have the Bochner integral (for totally mesaurable functions). When $E$ is not locally convex, $\mu$ is not always of bounded semivariation. But if $\mu$ is of bounded semivariation and is regular (as a positive measure), then as a vector measure $\mu \in M_{n}\left[L\left(E, E_{\sigma}^{\prime \prime}\right)\right]$.

Przeworska-Rolewicz and Rolewicz [16, p. 129] have shown that when $\mu$ is (Borel-)Lebesgue measure on $Q=[0,1]$ and $E=L^{p}[0,1], 0<p<1$, then the $E$-valued integral determined by $\mu$ is not continuous on $S(Q, E)$. Gramsch [9, p. 194] has shown that, for $Q=\left[0, \pi^{2} / 6\right]$ and $E=l^{1 / 3}$, not every $E$-valued continuous function is Riemann-integrable and a similar argument shows that this is true for Lebesgue measure. Thus, by (BSV) and Proposition 1, in these cases Lebesgue measure is not of bounded semivariation as an $L(E, E)$-valued set function. We have the following general necessary condition.

Theorem 8. If a finite positive Borel measure $\mu$ on the real line is of bounded semivariation for $l^{p}, 0<p<1$, then the mass points of $\mu$ are dense in the sup. port of $\mu$.

Proof. Suppose the contrary. The mass points of $\mu$ are the discontinuities of the distribution function $g(t)=\mu(-\infty, t]$, so the support of $\mu$ contains an interval $\left[a_{0}, b_{0}\right]$ on which $g$ is continuous and not constant. Let $S$ be the sum of the convergent series $\Sigma_{i}-1 / p$ and $b=\left[g\left(b_{0}\right)-g\left(a_{0}\right)\right] / S$. There is a sequence 
$\left(a_{i}\right)$ in $\left[a_{0}, b_{0}\right]$ such that, for each $i$,

$$
g\left(a_{i}\right)-g\left(a_{i-1}\right)=b i^{-1 / p}
$$

Let $n$ be a positive integer, $A_{0}=\left(-\infty, a_{0}\right], A_{i}=\left(a_{i-1}, a_{i}\right], i=1,2, \cdots, n$, and $A_{n+1}=\left(a_{n}, \infty\right)$. Let $x_{0}=x_{n+1}=0$ in $l^{p}$ and $x_{i}=\left\{\delta_{i j}\right\}, 1 \leq i \leq n$. Then

$$
\left\|\sum_{i=0}^{n+1} \mu\left(A_{i}\right) x_{i}\right\|=\sum\left[g\left(a_{i}\right)-g\left(a_{i-1}\right)\right]^{p}=b^{p} \sum_{i=1}^{n} i^{-1},
$$

which is unbounded in $n$, so we have a contradiction.

Corollary. On any bounded interval, Lebesgue measure is not of bounded semivariation in $l^{p}, 0<p<1$.

The next result gives a sufficient condition but places stringent restrictions on $\mu$.

Theorem 9. Let $\mu$ and $\lambda$ be finite positive Borel measures on $[0,1]$ such that $\mu^{p} \leq \lambda, 0<p<1$. Then $\mu$ is of bounded variation on every p-normed space $E$, has the properties of Theorem 6 and is singular with respect to Lebesgue mea. sure $m$.

Proof. The first two assertions follow from $\|\mu(A)\|=\mu(A)^{p}$.

Now let $g$ and $b$ be the distribution functions of $\mu$ and $\lambda$, respectively, $a \in(0,1)$ and $a<b \leq 1$. Then

$$
0 \leq g(b)-g(a) \leq[b(b)-b(a)]^{1 / p},
$$

so

$$
0 \leq \frac{g(b)-g(a)}{b-a} \leq \frac{b(b)-b(a)}{b-a}[b(b)-b(a)]^{(1 / p)-1} .
$$

Thus if $b_{+}^{\prime}(a)$ exists, then $g_{+}^{\prime}(a)=0$ and, similarly, if $b_{-}^{\prime}(a)$ exists, then $g_{-}^{\prime}(a)=0$. Since $b^{\prime}$ exists a.e. $[m]$, we have $g^{\prime}=0$ a.e. $[m]$ and the proof is complete.

We conclude with a method for constructing measures of the above type. Let $0<p<1, x=\left(b_{i}\right)$ be a positive sequence in $l^{p}$ and $\left(t_{i}\right)$ be an enumeration of of the rationals in $[0,1]$. Then $\mu(A)=\Sigma\left\{b_{i}: t_{i} \in A\right\}$ defines a positive Borel measure on $[0,1]$. By Jensen's inequality, $\Sigma b_{i} \leq\left[\Sigma\left(b_{i}\right)^{p}\right]^{1 / p}$, so $\mu$ is finite. But $\lambda(A)=\Sigma\left\{\left(b_{i}\right)^{p}: t_{i} \in A\right\}$ defines a finite positive Borel measure such that $\mu^{p} \leq \lambda$, so $\mu$ is of bounded variation on $l^{p}$ and is singular $[m]$.

Added in proof. In Corollaries 1 and 2 to Theorem 3, the representing measures are actually countably additive and regular for $L_{s}\left(E, F_{c}\right)$ where the subscript $s$ 
denotes the simple topology. They are clearly countably additive and regular for the topology induced by $L_{s}\left(E, F_{\sigma}\right)$, which is also the weak topology of $L_{s}\left(E, F_{c}\right)$ $[17$, p. 139]. The result now follows from Theorems 1.1 and 1.6 of D. R. Lewis, Integration with respect to vector measures, Pacific J. Math. 33 (1970), 157-165. MR $41 \# 3706$.

\section{REFERENCES}

1. N. Bourbaki, Éléments de mathématique. XXV. Part I. Livre VI: Intégration. Chap. 6: Intégration vectorielle, Actualités Sci. Indust., no. 1281, Hermann, Paris, 1959. MR 23\# A2033.

2. N. Colojoară, N. Dinculeanu and Gh. Marinescu, Measures with values in locally convex spaces, Bull. Math. Soc. Sci. Math. Phys. R. P. Roumaine 5 (53) (1961), 167-180. MR 31 \#6109.

3. N. Dinculeanu, Vector measures, Internat. Series of Monographs in Pure and Appl. Math., vol. 95, Pergamon Press, Oxford; VEB Deutscher Verlag der Wissenschaften, Berlin, 1967. MR $34 \# 6011$ b.

4. F. L. Duren, B. W. Romberg and A. L. Shields, Linear functionals on $H^{p}$ spaces with $0<p<1$, J. Reine Angew. Math. 238 (1969), 32-60. MR 41 \#217.

5. J. K. Edwards and S. G. Wayment, A V-integral representation for linear operators on spaces of continuous functions with values in topological vector spaces, Pacific J. Math. 35 (1970), 327-330. MR 43 \#465.

6. - Integral representations for continuous linear operators in the setting of convex topological vector spaces, Trans. Amer. Math. Soc. 157 (1971), 329-346.

7. C. Foias and I. Singer, Some remarks on the representation of linear operators in spaces of vector-valued continuous functions, Rev. Math. Pures Appl. 5 (1960), 729-752. MR 24 \#A 1618.

8. R. K. Goodrich, A Riesz representation theorem, Proc. Amer. Math. Soc. 24 (1970), 629-636.

9. B. Gramsch, Integration und holomorphe Funktionen in lokalbeschränkten Räumen, Math. Ann. 162 (1965/66), 190-210. MR 33 \#563.

10. - Tensorprodukte und Integration vektorwertiger Funktionen, Math. Z. 100 (1967), 106-122. MR $35 \# 7115$.

11. D. A. Gregory and J. H. Shapiro, Nonconvex linear topologies with the HahnBanach extension property, Proc. Amer. Math. Soc. 25 (1970), 902-905. MR 41 \#8957.

12. J. L. Kelley, I. Namioka et al., Linear topological spaces, University Series in Higher Math., Van Nostrand, Princeton, N. J., 1963. MR 29 \#3851.

13. S. Mazur and W. Orlicz, Sur les espaces métriques linéaires. I, II, Studia Math. 10 (1948), 184-208; ibid. 13 (1953), 137-179. MR 10, 611; MR 16, 932.

14. A. Pietsch, Nukleare lokalkonvexe Räume, Schriftenreihe der Institute für Mathematik bei der Deutschen Akademie der Wissenschaften zu Berlin, Reihe A, Reine Mathematik, Heft 1, Akademie-Verlag, Berlin, 1965. MR 31 \#114.

15. V. Popescu, Integration with respect to measures with values in arbitrary topological vector spaces, Stud. Cerc. Mat. 18 (1966), 1159-1180. MR 39 \#401.

16. D. Przeworska-Rolewicz and S. Rolewicz, On integrals of functions with values in a complete linear metric space, Studia Math. 26 (1966), 121-131. MR 33 \#564.

17. H. H. Schaefer, Topological vector spaces, Macmillan, New York 1966. MR 33 $\# 1689$.

18. A. H. Shuchat, Integral representation theorems in topological vector spaces, Dissertation, University of Michigan, Ann Arbor, Mich., 1969.

19. - Approximation of vector-valued continuous functions, Proc. Amer. Math. Soc. 31 (1972), 97-103. 
20. I. Singer, Linear functionals on the space of continuous mappings of a compact Hausdorff space into a Banach space, Rev. Math. Pures Appl. 2 (1957), 301-315. (Russian) MR 20 \#3445.

21. E. F. Steiner, On finite-dimensional linear topological spaces, Amer. Math. Monthly 72 (1965), 34-35. MR 30 \#1386.

22. K. Swong, A representation theory of continuous linear maps, Math. Ann. 155 (1964), 270-291; errata, ibid. 1157 (1964), 178. MR 29 \#2642.

23. B. L. D. Thorp, Equivalent notions of bounded variation, J. London Math. Soc. 43 (1968), $247-252$.

24. P. Turpin and L. Waelbroeck, Sur l'approximation des fonctions différentiables á valeurs dans les espaces vectoriels topologiques, C. R. Acad. Sci. Paris Sér. A-B 267 (1968), A94-A97. MR 38 \#2597.

25. M. P. Ulanov, Vector-valued set functions and the representation of continuous linear mappings, Sibirsk. Mat. Ž. 9 (1968), 410-425. (Russian) MR 37 \#46.

26. D. Vogt, Integrationstheorie in p-normierten Räumen, Math. Ann. 173 (1967), 219-232. MR $36 \# 345$.

27. C. Swartz, Absolutely summing and dominated operators on spaces of vectorvalued continuous functions (to appear).

DEPARTMENT OF MATHEMATICS, UNIVERSITY OF TOLEDO, TOLEDO, OHIO 43606

Current address: Department of Mathematics, Mount Holyoke College, South Hadley, Massachusetts 01075 